\title{
HIGHER DIMENSIONAL EXPANDING MAPS AND TORAL EXTENSIONS
}

\author{
EUGEN MIHAILESCU
}

(Communicated by Bryna Kra)

\begin{abstract}
We prove that expanding endomorphisms on arbitrary tori are 1-sided Bernoulli with respect to their corresponding measure of maximal entropy and are thus, measurably, as far from invertible as possible. This applies in particular to expanding linear toral endomorphisms and their smooth perturbations. Then we study toral extensions of expanding toral endomorphisms, in particular probabilistic systems on skew products, and prove that under certain not too restrictive conditions on the extension cocycle, these skew products are 1-sided Bernoulli too. We also give a large class of examples of group extensions of expanding maps in higher dimensions, for which we check the conditions on the extension cocycle.
\end{abstract}

\section{INTRODUCTION AND OUtLINE OF MAIN RESUlts}

From the point of view of ergodic properties, endomorphisms of Lebesgue spaces behave in a very different way from automorphisms. The study of measurepreserving endomorphisms also presents many different methods and ideas than that of diffeomorphisms/automorphisms. Even for 1-sided Bernoulli shifts, the problem of classification up to measure-theoretic isomorphism is very far from the similar problem for 2-sided Bernoulli maps. For 2-sided Bernoulli shifts, it is well known that Ornstein showed they can be classified by measure-theoretic entropy alone (see [16], 26]), while for 1-sided shifts this is not at all the case. In fact, as Parry and Walters (21], 26]) showed, endomorphisms $T$ on Lebesgue spaces $(X, \mathcal{B}, \mu)$ cannot be classified even by a combination of entropy, Jacobian and the sequence of decreasing algebras $\left\{T^{-n} \mathcal{B}\right\}_{n \geq 0}$.

The existence of multiple preimages of a point and the possibly different behaviors of consecutive sums on different prehistories of points imply that the dynamical and ergodic properties of endomorphisms are different in results and in techniques than those of automorphisms.

Endomorphisms were studied under various aspects, both from the point of view of smooth dynamical behavior in the expanding/hyperbolic case, as well as from the point of view of ergodic/statistical properties by several authors; see for instance [2], 3], 4], [5], 6], 7], 8], 11, [18, 21], 24], 25], 13], 14], 15], etc.

Received by the editors November 14, 2011 and, in revised form, December 10, 2011.

2010 Mathematics Subject Classification. Primary 37D20, 37A35, 37C40.

Key words and phrases. Toral endomorphisms, expanding maps, 1-sided Bernoullicity, measures of maximal entropy, group extensions, cohomological conditions.

This work was supported by CNCS - UEFISCDI, project PN II - IDEI PCE 2011-3-0269. 
Ashley, Marcus and Tuncel gave in [2] a complete and computable classification of 1-sided Markov chains (hence in particular of 1-sided Bernoulli shifts) up to measure-theoretic isomorphism. In [8], Katznelson proved that an automorphism of the $m$-dimensional torus $\mathbb{T}^{m}, m \geq 2$, whose eigenvalues are never roots of unity, must be a 2-sided Bernoulli shift. However, in the case of an endomorphism $f_{A}$ of $\mathbb{T}^{m}$ induced by the matrix $A$, the situation is completely different, and we cannot say a priori whether $f_{A}$ is 1-sided Bernoulli with respect to the corresponding Lebesgue (Haar) measure. Also in 8 it was shown that the inverse limit (natural extension) of a hyperbolic toral endomorphism is 2-sided Bernoulli; however this does not imply that the endomorphism $f_{A}$ is 1-sided Bernoulli. In [6] there are examples of endomorphisms $T$ which are uniformly 2-to-1 but which are not 1 sided Bernoulli, although the square $T^{2}$ is 1-sided Bernoulli. Thus the inverse limit $S^{2}$ of $T^{2}$ is 2-sided Bernoulli and, from Ornstein [16, the inverse limit $S$ (of $T$ ) is 2-sided Bernoulli.

The main results of the paper are:

In Theorem 1 we show that an arbitrary expanding (linear) toral endomorphism $f_{A}$ is 1-sided Bernoulli with respect to the Haar measure by using a result of Hoffman and Rudolph from [7].

In Corollary 1 we extend Theorem 1 to smooth expanding endomorphisms $g$ on tori (not necessarily linear), together with their respective measure of maximal entropy $\mu_{0, g}$ on $\mathbb{T}^{m}$; in particular, this applies to smooth perturbations of expanding linear toral endomorphisms. We notice that there is a relation between dilation and 1-sided Bernoullicity.

We then study group extensions for expanding toral endomorphisms $f_{A}$, given by weakly mixing skew products associated to summable cocycles with values in tori. These are non-invertible maps $f_{A, \psi}: \mathbb{T}^{m} \times \mathbb{T}^{k} \rightarrow \mathbb{T}^{m} \times \mathbb{T}^{k}$ of type

$$
f_{A, \psi}\left(x, y_{1}, \ldots, y_{k}\right)=\left(f_{A}(x), \psi_{1}(x)+y_{1}(\bmod 1), \ldots, \psi_{k}(x)+y_{k}(\bmod 1)\right),
$$

with $x \in \mathbb{T}^{m},\left(y_{1}, \ldots, y_{k}\right) \in \mathbb{T}^{k}$ and where $\psi: \mathbb{T}^{m} \rightarrow \mathbb{T}^{k}$ is a Hölder continuous function. For generalities about group extensions, see for instance [1, 4], 18, [20], etc. The conditions for a toral extension $f_{A, \psi}$ to be weakly mixing with respect to the product of Haar measures on $\mathbb{T}^{m}$ and $\mathbb{T}^{k}$, are related to the "linear independence" of the components of the cocycle or, in other words, to the fact that $\psi$ is not a coboundary (see [1, 19], 20, etc.).

In Theorem 2 we find a large class of examples of group extensions of higher dimensional expanding toral endomorphisms which are shown to be 1sided Bernoulli as well. Such toral extensions can also be constructed for perturbations $g$ of $f_{A}$, as above.

In particular, for expanding toral endomorphisms and for extensions as above, there will exist generating Rohlin partitions (see [5]).

\section{1-Sided Bernoulli toral maps. Group eXtensions}

In the sequel we work with Lebesgue space systems, i.e. with measurable endomorphisms $f: X \rightarrow X$ on Lebesgue spaces $(X, \mathcal{B}, \mu)$ s.t. $f$ preserves the probability measure $\mu$. In some cases, when there is no confusion on the $\sigma$-algebra $\mathcal{B}$ on $X$, we shall write only $(X, \mu)$ for the Lebesgue space and $(X, \mu, f)$ for the system. We shall investigate toral endomorphisms of type $f_{A}: \mathbb{T}^{m} \rightarrow \mathbb{T}^{m}, m \geq 2$, given by an integer-valued matrix $A$, all of whose eigenvalues are strictly larger 
than 1 in absolute value. Then we see that $f_{A}$ is a distance expanding map with respect to the Riemannian metric on $\mathbb{T}^{m}$. We consider the Lebesgue (Haar) measure $\mu_{m}$ on $\mathbb{T}^{m}$, which clearly is preserved by $f_{A}$.

A 1-sided Bernoulli shift is a Lebesgue space system $(X, \mu, f)$ isomorphic to the model system $\left(\Sigma_{r}^{+}, \sigma_{r}, \nu_{p}\right)$, where $\Sigma_{r}^{+}=\left\{\left(\omega_{0}, \omega_{1}, \ldots\right), \omega_{j} \in\{1, \ldots, r\}, j \geq 0\right\}$ is the space of positively indexed sequences on $r$ symbols and where $\sigma_{r}, \nu_{p}$ are as before. A uniform model 1-sided Bernoulli shift $\left(\Sigma_{r}^{+}, \nu_{\left(\frac{1}{r}, \ldots, \frac{1}{r}\right)}, \sigma_{r}\right)$ corresponds to the uniform probability vector $\mathbf{p}$ with $p_{i}=\frac{1}{r}, i=1, \ldots, r$.

As said before, ergodic properties of 1-sided Bernoulli shifts are very different from those for 2-sided Bernoulli shifts, due to non-invertibility ([21], 26], etc.). The notion of very weakly Bernoulli (for instance [9], 8, etc.) involves automorphisms which interchange the sets in partitions of type $T^{k} \xi, k \geq 1$ (for a certain partition $\xi)$; it is a generalization of weakly Bernoulli (see [3], [17, etc.).

Consider $f: X \rightarrow X$ a measure-preserving endomorphism on a Lebesgue space $(X, \mathcal{B}, \mu)$, and let the measurable partition of $X$ be given by fibers $f^{-1}(x), x \in X$. Associated to this partition there is the family of conditional measures of $\mu$, denoted by $\left\{\mu_{x}\right\}_{x}($ see $[22])$.

In [7, Hoffman and Rudolph introduced a notion of tree very weakly Bernoulli for endomorphisms, which we now recall. Assume $f$ is uniformly $r$-to-one, i.e. that $h_{\mu}(f)=\log r$, where $x \in X$ has $r f$-preimages and the conditional measures $\mu_{x}$ are uniformly distributed in $f^{-1}(x)$, for $\mu$-a.a. $x \in X$. Let $\mathcal{T}$ be an abstract infinite tree having $r^{n}$ nodes at each index (level) $n \geq 0$ and $\mathcal{T}_{n}$ be the truncation at level $n$ of $\mathcal{T}$. Now for $Y$ a compact metric space, a $(\mathcal{T}, Y)$-name is any function $h: \mathcal{T} \rightarrow Y$; $h$ is tree adapted if for any $v$ and $i, j \in\{0, \ldots, r-1\}, i \neq j$, we have $h(v i) \neq h(v j)$. If $\phi: X \rightarrow Y$ is a map, let $\mathcal{F}(\phi)$ be the $\sigma$-algebra on $X$ given by the pullback of borelians $\mathcal{B}(Y)$ through $\phi$; we say $\phi$ generates if $\bigvee_{i} f^{-i}(\mathcal{F}(\phi))=\mathcal{B}$. Next take a Rohlin partition $\left\{E_{0}, \ldots, E_{r-1}\right\}$ of $(X, \mathcal{B}, \mu)$ (see [22], [3]) s.t. $\left.f\right|_{E_{i}}: E_{i} \rightarrow X$ is bijective $\mu$-a.e., and let $f_{i}$ be the inverse of $\left.f\right|_{E_{i}}, i$. For a node $v \in \mathcal{T}_{n}$, define the inverse iterate $f_{v}(x):=f_{j_{n}}\left(\ldots\left(f_{j_{1}}(x)\right) \ldots\right)$. For $x \in X$ the $(\mathcal{T}, Y)$-name $\mathcal{T}_{x}^{\phi}$ of $\phi$-values on the preimages of $x$ is given by $\mathcal{T}_{x}^{\phi}(v):=\phi\left(f_{v}(x)\right), v \in \mathcal{T}$. A map $\phi: X \rightarrow Y$ is called tree adapted on $X$ if $\mathcal{T}_{x}^{\phi}$ is a tree adapted $(\mathcal{T}, Y)$-name for $\mu$-a.e. $x \in X$. Let $\mathcal{A}$ be the set of tree automorphisms on $\mathcal{T}$ and $\mathcal{A}_{n}$ the similar set on $\mathcal{T}_{n}$. For $n>1, t_{n}(g, h):=\inf _{A \in \mathcal{A}_{n}} \frac{1}{n} \sum_{0<|v| \leq n} \frac{1}{r^{|v|}} d(h(v), g(A v))$ gives a metric on $(\mathcal{T}, Y)$-names. Then $(X, \mathcal{B}, \mu, f)$ and the tree adapted map $\phi: X \rightarrow Y$ are called tree very weakly Bernoulli if, for any $\varepsilon>0$ and $n$ large enough, there exists a set $G(\varepsilon, n)$ with $\mu(G(\varepsilon, n))>1-\varepsilon$ s.t. $t_{n}\left(\mathcal{T}_{z}^{\phi}, \mathcal{T}_{w}^{\phi}\right)<\varepsilon, \forall z, w \in G(\varepsilon, n)$.

Theorem 1. Let $f_{A}$ be a toral endomorphism on $\mathbb{T}^{m}, m \geq 2$, given by the integervalued matrix A, all of whose eigenvalues are strictly larger than 1 in absolute value. Then the endomorphism $f_{A}$ on the torus $\mathbb{T}^{m}$ equipped with its Lebesgue (Haar) measure $\mu_{m}$ is isomorphic to a uniform model 1-sided Bernoulli shift.

Proof. From the fact that all the eigenvalues of $A$ are larger than 1 in absolute value, it follows that $f_{A}$ is an expanding map on $\mathbb{T}^{m}$. Also it is well-known that $f_{A}$ is $|\operatorname{det}(A)|$-to-1 on $\mathbb{T}^{m}$ (see for instance [26]). Assume $|\operatorname{det}(A)|=r \geq 2$ (otherwise $f_{A}$ is an automorphism, and toral automorphisms are 2-sided Bernoulli shifts by [8]). 
Now if $f_{A}$ is expanding and $r$-to- 1 , we know that $\mu_{m}$ is the limit of a sequence of probability measures of type

$$
\nu_{n}^{x}:=\frac{1}{r^{n}} \sum_{y \in f_{A}^{-n} x} \delta_{y},
$$

for some $x \in \mathbb{T}^{m}$ (see for example [24, 10]). Thus by working for $\nu_{n}^{x}$, then going to limit $(n \rightarrow \infty)$ on borelian sets with boundaries of $\mu_{m}$-measure zero, and finally by approximating arbitrary borelians with such sets, we obtain that

$$
\mu_{m}\left(f_{A}(B)\right)=r \mu_{m}(B)
$$

for any borelian set $B$ so that $\left.f\right|_{B}$ is injective. Thus the conditional probabilities of $\mu_{m}$, associated to the partition $\xi$ into fibers $\left\{f^{-1}(z)\right\}_{z}$, are equidistributed on the fibers of $\mu_{m}$-almost all points from $\mathbb{T}^{m}$; i.e. they give weight equal to $\frac{1}{r}$ to each of the $r$ preimages of $z$.

But $\mu_{m}$ is the Lebesgue (Haar) measure on $\mathbb{T}^{m}$ and $f_{A}$ is supposed to be $r$ to- 1 , hence the entropy $h_{\mu_{m}}\left(f_{A}\right)=\log r$. So $h_{\mu_{m}}\left(f_{A}\right)=\log r$, the conditional probabilities of $\mu_{m}$ on the preimages are all equal to $\frac{1}{r}$ and $f_{A}$ is $r$-to- 1 , meaning that $\left(\mathbb{T}^{m}, \mathcal{B}, \mu_{m}, f_{A}\right)$ is a uniform measure-preserving endomorphism.

Now $f_{A}$ is expanding and open (since $\left.f\right|_{\Lambda}$ is $r$-to-1); hence $\left.f\right|_{\Lambda}$ is topologically exact. Thus for any $\varepsilon>0$ small there exists some positive integer $N$ (independent of $y, z)$ so that, given any $y, z \in \mathbb{T}^{m}$ and any $N$-preimage $y_{-N}$ of $y$, there exists an $N$-preimage $z_{-N}$ of $z$ such that

$$
d\left(y_{-N}, z_{-N}\right)<\varepsilon .
$$

As our generating function we will take the identity $I d: \mathbb{T}^{m} \rightarrow \mathbb{T}^{m}$, which clearly generates the $\sigma$-algebra of borelians on $\mathbb{T}^{m}$.

From (11) and the fact that local inverse iterates of $f$ contract distances, we infer that given any points $y, z \in \mathbb{T}^{m}$, there exists $N=N(\varepsilon)$ such that for any $n>N$ and any $n$-preimage $y_{-n}$ of $y$, there exists a unique $n$-preimage $z_{-n}$ of $z$ so that $z_{-n} \in B_{n}\left(y_{-n}, \varepsilon\right)$. Vice-versa, for any $n$-preimage $z_{-n} \in \Lambda$ of $z$, there is a unique $n$-preimage $y_{-n} \in \Lambda$ of $y$ with $y_{-n} \in B_{n}\left(z_{-n}, \varepsilon\right)$. Therefore for any $\varepsilon>0$, there exists $N(\varepsilon)$ so that we have

$$
t_{n}\left(\mathcal{T}_{y}, \mathcal{T}_{z}\right)<C \varepsilon, \forall y, z \in \mathbb{T}^{m}, n>N(\varepsilon),
$$

where $C>0$ is a constant independent of $\varepsilon, n, y, z(C$ depends only on the minimum expansion coefficient of $f_{A}$ on $\left.\mathbb{T}^{m}\right)$. So in our case the set $G(\varepsilon, n)$ from the definition of tree very weakly Bernoulli is the whole $\mathbb{T}^{m}$.

Thus the measure-preserving uniform endomophism $\left(\mathbb{T}^{m}, \mathcal{B}, \mu_{m}, f_{A}\right)$ and the generating function $I d: \mathbb{T}^{m} \rightarrow \mathbb{T}^{m}$ are tree very weakly Bernoulli.

In conclusion, from the characterization result for 1-sided Bernoulli shifts given in [7], we see that $\left(\mathbb{T}^{m}, \mathcal{B}, \mu_{m}, f_{A}\right)$ is 1-sided Bernoulli and conjugate to the uniform model $\left(\Sigma_{r}^{+}, \nu_{\left(\frac{1}{r}, \ldots, \frac{1}{r}\right)}, \sigma_{r}\right)$.

We now consider arbitrary expanding maps $g: \mathbb{T}^{m} \rightarrow \mathbb{T}^{m}$, i.e maps with $\|D g\|>1$ on $\mathbb{T}^{m}$. A particular case is when $g$ is a smooth perturbation of an expanding linear toral endomorphism.

Corollary 1. Let $g$ be an expanding endomorphism on $\mathbb{T}^{m}$, and denote by $\mu_{0, g}$ its unique measure of maximal entropy. Then the system $\left(\mathbb{T}^{m}, \mu_{0, g}, g\right)$ is 1-sided 
Bernoulli. In particular, this applies when $g$ is a smooth perturbation of an expanding linear endomorphism of $\mathbb{T}^{m}$.

Proof. An expanding map $g$ has a unique measure of maximal entropy $\mu_{0, g}$. We know that $\mu_{0, g}$ is absolutely continuous with respect to the Lebesgue measure $\mu_{m}$ and that the Radon-Nykodim derivative $\frac{d \mu_{0, g}}{d \mu_{m}}$ is Hölder continuous and bounded away from 0 and $\infty$ (see for instance 10]).

Since $g$ is an expanding endomorphism on $\mathbb{T}^{m}$, it follows from Shub (see [25]) that $g$ is topologically conjugate to a linear endomorphism $f_{A}$ on $\mathbb{T}^{m}$. Thus there exists a homeomorphism $H: \mathbb{T}^{m} \rightarrow \mathbb{T}^{m}$ so that $H \circ g=f_{A} \circ H$. This implies that since $f_{A}$ is $r$-to- 1 (where $r=|\operatorname{det} A|$ ), then $g$ is also $r$-to- 1 on $\mathbb{T}^{m}$. Also, we obtain that the topological entropy of $g$ is the same as the topological entropy of $f_{A}$, i.e. $h_{\text {top }}(g)=h_{\mu_{0, g}}(g)=h_{\text {top }}\left(f_{A}\right)=\log r$.

Next, if $g$ is expanding we can apply the results of [10, 24] in order to obtain the unique measure of maximal entropy $\mu_{0, g}$ of $g$ as the limit of the sequence of probabilities

$$
\nu_{g, n}^{x}:=\frac{\sum_{y \in g^{-n}(x)} \delta_{y}}{r^{n}}, n \geq 1 .
$$

But as in the proof of Theorem 1 this shows that $\mu_{0, g}(g(B))=r \mu_{0, g}(B)$, for any borelian set $B \subset \mathbb{T}^{m}$. Thus the conditional measures of $\mu_{0, g}$ associated to the fiber partition are equally distributed among the $r$ preimages in almost all fibers of $g$. Thus we obtain that $g$ is a uniform $r$-to- 1 endomorphism with respect to the measure $\mu_{0, g}$ on $\mathbb{T}^{m}$.

Now we proceed as in the proof of Theorem 1 in order to obtain that $g$ is tree very weakly Bernoulli and thus that the system $\left(\mathbb{T}^{m}, \mu_{0, g}, g\right)$ is 1 -sided Bernoulli.

For the particular case, if $g$ is a smooth perturbation of an expanding linear toral endomorphism, then it is clear that $g$ itself is expanding and we can apply the above arguments.

Example. An example of a perturbation of an expanding toral endomorphism is $g: \mathbb{T}^{2} \rightarrow \mathbb{T}^{2}:$

$$
g(x, y)=(2 x+\varepsilon \sin (2 \pi x+4 \pi y), 3 y+\varepsilon \cos (2 \pi x)),(x, y) \in \mathbb{T}^{2} .
$$

The expanding map $g$ has a unique measure of maximal entropy $\mu_{0, g}$ on $\mathbb{T}^{2}$, and this measure is absolutely continuous with respect to the Haar measure, although not necessarily equal to it. So from Corollary 19 is 1-sided Bernoulli with respect to its measure of maximal entropy $\mu_{0, g}$.

Corollary 2. Let the hyperbolic linear toral endomorphism $f_{A}$ on $\mathbb{T}^{m}$ with $|\operatorname{det}(A)|$ $>1$. Then $f_{A}$ is 1-sided Bernoulli with respect to the Haar measure $\mu_{m}$ on $\mathbb{T}^{m}$ if and only if $f_{A}$ is expanding; i.e. all the eigenvalues of $A$ are strictly larger than 1 in absolute value.

Proof. The proof follows from Theorem 1 above and from Theorem 2 and Corollary 1 of $[12$.

If $f_{A}$ is expanding and $\mu_{\phi}$ is the equilibrium measure of a Hölder potential $\phi$, then it also follows that $\left(\mathbb{T}^{m}, \mu_{\phi}, f_{A}\right)$ is 1-sided Bernoulli if and only if $\mu_{\phi}$ is the Haar measure $\mu_{m}$ (see [12]). 
We now study a special class of endomorphisms, namely group extensions, which are skew-products that can be constructed starting with known endomorphisms. Many aspects of group extensions have been investigated in the literature, for example in [1, 4], 18, 19, 20, 23, etc.

Let us start with a measure-preserving endomorphism $f$ on a Lebesgue space $f$ : $(X, \mathcal{B}, \mu) \rightarrow(X, \mathcal{B}, \mu)$. Also consider a compact metric space $(Z, d)$ with $\operatorname{Isom}(Z)$ to be the space of its isometries (with uniform topology). Assume that $\operatorname{Isom}(Z)$ acts transitively on $Z$, so $Z$ is a homogeneous space. Then $Z$ is homeomorphic to $\operatorname{Isom}(Z) / H$ for some closed subgroup $H \subset I \operatorname{Isom}(Z)$. Now we can consider on $Z$ the restricted Haar measure $\mu_{Z}$ induced from the topological group with uniform topology $G=I \operatorname{som}(Z)$ (see [23]). Next let us take an arbitrary function $\psi: X \rightarrow \operatorname{Isom}(Z)$ and define the group extension $f_{\psi}: X \times Z \rightarrow X \times Z$,

$$
f_{\psi}(x, z)=(f(x), \psi(x)(z)),(x, z) \in X \times Z .
$$

The function $\psi$ is called a cocycle and $f_{\psi}$ a cocycle extension. On $X \times Z$ we consider the product measure $\mu \times \mu_{Z}$, where $\mu_{Z}$ is the induced Haar measure on $Z$. The cocycle $\psi$ is called a coboundary with respect to the endomorphism $f: X \rightarrow X$ if there exists a measurable function $\chi$ and a constant $c$ so that $\psi=\chi \circ f-\chi+c$, $\mu$-almost everywhere.

In the sequel we shall work with a specific case, namely when the metric space $Z$ is a torus $\mathbb{T}^{k}, k \geq 1$. We will use the additive notation on $\mathbb{T}^{k}$. Our cocycle will be given by a map $\psi: \mathbb{T}^{m} \rightarrow \mathbb{T}^{k}, \psi=\left(\psi_{1}, \ldots, \psi_{k}\right)$, with $\psi_{i}: \mathbb{T}^{m} \rightarrow S^{1}, i=$ $1, \ldots, k$. The group extension of the expanding toral endomorphism $f_{A}: \mathbb{T}^{m} \rightarrow \mathbb{T}^{m}$ is the skew product $f_{A, \psi}: \mathbb{T}^{m} \times \mathbb{T}^{k} \rightarrow \mathbb{T}^{m} \times \mathbb{T}^{k}, f_{A, \psi}(x, z)=\left(f_{A}(x), \psi_{1}(x)+\right.$ $\left.z_{1}(\bmod 1), \ldots, \psi_{k}(x)+z_{k}(\bmod 1)\right),(x, z) \in \mathbb{T}^{m} \times \mathbb{T}^{k}$, which preserves the product measure $\mu_{m} \times \mu_{k}$ on $\mathbb{T}^{m} \times \mathbb{T}^{k}$ (where $\mu_{m}$ and $\mu_{k}$ represent the Lebesgue (Haar) measures on $\mathbb{T}^{m}$, respectively on $\left.\mathbb{T}^{k}\right)$.

Now let us assume that the map $\psi: \mathbb{T}^{m} \rightarrow \mathbb{T}^{k}$ used above is Hölder continuous. Since $f_{A}$ is distance expanding, we see that the branches of inverse iterates of $f_{A}$ contract exponentially. Hence from the Hölder continuity of $\psi$ it follows that $\psi$ is a summable cocycle.

The next step will be to assure the weak mixing of $f_{A, \psi}$ with respect to $\mu_{m} \times$ $\mu_{k}$. Ergodicity of a measure-preserving endomorphism $f$ means that the operator $U_{f}$, given by the composition with $f$ on integrable functions, has only constants as eigenfunctions of the eigenvalue 1 (26]). If $f$ is ergodic and, in addition, $U_{f}$ has no eigenfunctions except essential constants, then $f$ has continuous spectrum. However, this is equivalent to weak mixing (see for instance [26]); thus there are strong relations between weak mixing and Livsic type conditions.

Criteria for the weak mixing of the group extension endomorphism $f_{\psi}$ were given first for skew products with rotations ([1]) and then in an abstract setting (see for instance [19], 20], 23], etc.), and they are centered on the condition that $\psi$ is not a coboundary.

Assume that $\psi$ is Hölder continuous with exponent $\alpha>0$, i.e. $\psi \in \mathcal{C}^{\alpha}\left(\mathbb{T}^{m}, \mathbb{T}^{k}\right)$. Then we have

Theorem (Mixing conditions for extensions, 20]). The above expanding map $f_{A, \psi}$ is weak mixing with respect to the product of Haar measures $\mu_{m} \times \mu_{k}$ on $\mathbb{T}^{m} \times \mathbb{T}^{k}$ if the equation

$$
F \circ f_{A}(x)=c+\ell_{1} \psi_{1}(x)+\ldots+\ell_{k} \psi_{k}(x)+F(x) \bmod 1, \text { a.e. },
$$


with $F: \mathbb{T}^{m} \rightarrow \mathbb{R}$ measurable, $\left(\ell_{1}, \ldots, \ell_{k}\right) \in \mathbb{Z}^{k}$ and $c \in \mathbb{R}$, has only the trivial solution $c=0,\left(\ell_{1}, \ldots, \ell_{k}\right)=(0, \ldots, 0)$ and $F$ constant.

Proposition 1. Let $f_{A}: \mathbb{T}^{m} \rightarrow \mathbb{T}^{m}$ be an expanding toral endomorphism and $\psi: \mathbb{T}^{m} \rightarrow \mathbb{T}^{k}$ be a Hölder continuous function. Assume that if there exist a measurable function $F$ on $\mathbb{T}^{m}$, a constant $c \in \mathbb{R}$ and a $k$-tuple of integers $\left(\ell_{1}, \ldots, \ell_{k}\right)$ with $F \circ f_{A}(x)=c+\ell_{1} \psi_{1}(x)+\ldots+\ell_{k} \psi_{k}(x)+F(x)(\bmod 1)$ a.e., then $c=0,\left(\ell_{1}, \ldots, \ell_{k}\right)=$ $(0, \ldots, 0)$ and $F$ is constant (i.e. equation (3) has only the trivial solution). Then the skew product $f_{A, \psi}$ is 1-sided Bernoulli with respect to the product of the respective Haar measures $\mu_{m} \times \mu_{k}$ on $\mathbb{T}^{m} \times \mathbb{T}^{k}$.

Proof. We know from the Hölder continuity of $\psi$ and from the uniform dilation of $f_{A}$ on $\mathbb{T}^{m}$ that $\psi$ generates a summable cocycle with respect to $f_{A}$.

Also, since the only solution to equation (3) is the trivial one, we obtain from the above mixing conditions for extensions that $f_{A, \psi}$ is weak mixing with respect to the product measure $\mu_{m} \times \mu_{k}$.

On the other hand, we showed in Theorem 1 that the expanding toral endomorphism $f_{A}$ is 1 -sided Bernoulli with respect to $\mu_{m}$.

Thus we can use Theorem 6.4 of [7] to conclude that the extension $f_{A, \psi}$ is tree very weakly Bernoulli, hence 1-sided Bernoulli with respect to $\mu_{m} \times \mu_{k}$.

The next theorem shows that there is a dense set of cocycles that give weak mixing extensions with expanding toral endomorphisms, and it also gives concrete examples where this happens.

Theorem 2. a) Let $f_{A}: \mathbb{T}^{m} \rightarrow \mathbb{T}^{m}$ be an expanding toral endomorphism and $\psi: \mathbb{T}^{m} \rightarrow \mathbb{T}^{k}$ be a Hölder continuous function from $\mathcal{C}^{\alpha}\left(\mathbb{T}^{m}, \mathbb{T}^{k}\right)$. Assume that there exists no constant $c$ s.t. for any periodic point $z \in \mathbb{T}^{m}$ with $f_{A}^{n}(z)=z$, $-n c=S_{n}\left(\ell_{1} \psi_{1}+\ldots+\ell_{k} \psi_{k}\right)(z) \bmod 1$, where $S_{n} \omega(y):=\omega(y)+\ldots+\omega\left(f_{A}^{n-1}(y)\right), y \in$ $\mathbb{T}^{m}, n \geq 1$. Then the group extension $f_{A, \psi}$ is 1 -sided Bernoulli with respect to the Lebesgue measure on $\mathbb{T}^{m} \times \mathbb{T}^{k}$.

b) The collection of cocycles $\psi \in \mathcal{C}^{\alpha}\left(\mathbb{T}^{m}, \mathbb{T}^{k}\right)$ which are not coboundaries in the sense of equation (3) (i.e. which give a 1-sided Bernoulli extension $\left.f_{A, \psi}\right)$, contains a dense $G_{\delta}$ set in the space $\mathcal{C}^{\alpha}\left(\mathbb{T}^{m}, \mathbb{T}^{k}\right)$ endowed with the norm $\|\psi\|:=|\psi|_{\alpha}+|\psi|_{\infty}$, where $|\psi|_{\alpha}:=\sup _{x \neq y} \frac{|\psi(x)-\psi(y)|}{|x-y|^{\alpha}}$ and $|\psi|_{\infty}$ is the uniform norm.

c) Consider the toral endomorphism $f_{A}$ given by the matrix $A=\left(\begin{array}{ll}2 & 1 \\ 0 & 6\end{array}\right)$, and the cocycle $\psi: \mathbb{T}^{2} \rightarrow \mathbb{T}^{2}$ given in additive notation by

$$
\psi\left(x_{1}, x_{2}\right)=\left(\sin 2 \pi\left(x_{1}+3 x_{2}\right), \sin 2 \pi x_{2}\right) .
$$

Then the extension $f_{A, \psi}$ is 1-sided Bernoulli with respect to the Lebesgue measure on $\mathbb{T}^{4}$.

Proof. a) Given $\psi=\left(\psi_{1}, \ldots, \psi_{k}\right) \in \mathcal{C}^{\alpha}\left(\mathbb{T}^{m}, \mathbb{T}^{k}\right)$, we know from Livsic results that there exists a measurable function $F: \mathbb{T}^{m} \rightarrow \mathbb{R}$ and a constant $c$ such that $F \circ$ $f_{A}(x)=c+\ell_{1} \psi_{1}(x)+\ldots+\ell_{k} \psi_{k}(x)+F(x) \bmod 1$ a.e. if and only if there exists $\tilde{F} \in \mathcal{C}^{\alpha}\left(\mathbb{T}^{m}, \mathbb{R}\right)$ such that $\tilde{F}(\cdot)=F(\cdot)$ a.e. and $\tilde{F} \circ f_{A}(x)=c+\ell_{1} \psi_{1}(x)+\ldots+$ $\ell_{k} \psi_{k}(x)+\tilde{F}(x) \bmod 1$, for all $x \in \mathbb{T}^{m}$ (see for instance [20]).

This last condition happens if and only if, for any periodic point $z \in \mathbb{T}^{m}$ with $f_{A}^{n}(z)=z$, we have that

$$
-n c=S_{n}\left(\ell_{1} \psi_{1}+\ldots+\ell_{k} \psi_{k}\right)(z) \bmod 1,
$$


where $S_{n} \omega(y):=\omega(y)+\ldots+\omega\left(f_{A}^{n-1}(y)\right), y \in \mathbb{T}^{m}$, defines the $n$-th consecutive sum of $\omega(\cdot)$, for $n \geq 1$.

b) From [19] it follows that the collection of functions $\psi \in \mathcal{C}^{\alpha}\left(\mathbb{T}^{m}, \mathbb{T}^{k}\right)$ which are not coboundaries in the sense of equation (3), i.e. which give a weak mixing extension $f_{A, \psi}$, contains a dense $G_{\delta}$ set in $\mathcal{C}^{\alpha}\left(\mathbb{T}^{m}, \mathbb{T}^{k}\right)$. This can be proved by using the above condition on periodic points.

Thus for "most" cocycles $\psi \in \mathcal{C}^{\alpha}\left(\mathbb{T}^{m}, \mathbb{T}^{k}\right)$, the toral extension $f_{A, \psi}$ is 1-sided Bernoulli with respect to the product of Haar measures $\mu_{m} \times \mu_{k}$.

c) First notice that the matrix $A$ has two eigenvalues larger than 1 , so $f_{A}$ is expanding on $\mathbb{T}^{2}$. With the toral endomorphism $f_{A}$ and the extension cocycle $\psi$ given above (which is well-defined on $\mathbb{T}^{2}$ ), we can form the toral extension $f_{A, \psi}$ : $\mathbb{T}^{2} \times \mathbb{T}^{2} \rightarrow \mathbb{T}^{2} \times \mathbb{T}^{2}$

$$
f_{A, \psi}\left(x_{1}, x_{2}, y_{1}, y_{2}\right)=\left(2 x_{1}+x_{2}, 6 x_{2}, y_{1}+\sin 2 \pi\left(x_{1}+3 x_{2}\right), y_{2}+\sin 2 \pi x_{2}\right) \bmod 1 .
$$

Let us check if condition (3) is satisfied. Assume there exists $\left(\ell_{1}, \ell_{2}\right) \in \mathbb{Z}^{2}$, a Hölder continuous function $F$ and a constant $c$ such that $F \circ f_{A}=c+\ell_{1} \psi_{1}+\ell_{2} \psi_{2}+$ $F(\bmod 1)$. Then

$$
-n c=S_{n}\left(\ell_{1} \psi_{1}+\ell_{2} \psi_{2}\right)(z) \bmod 1 \text { as long as } f_{A}^{n}(z)=z, n \geq 1
$$

But one of the fixed points of $f_{A}$ is $(0,0)$, so if $\ell_{1} \psi_{1}(0,0)+\ell_{2} \psi_{2}(0,0)=0$, then $c=0$. Now consider $\left(\frac{4}{5}, \frac{1}{5}\right)$, which is another fixed point of $f_{A}$. Then we should have

$$
\ell_{1} \psi_{1}\left(\frac{4}{5}, \frac{1}{5}\right)+\ell_{2} \psi_{2}\left(\frac{4}{5}, \frac{1}{5}\right)=0 \bmod 1 .
$$

This implies that $-\ell_{1} \sin \frac{\pi}{5}+\ell_{2} \sin \frac{2 \pi}{5}=0 \bmod 1$, but $\sin \frac{\pi}{5}=\frac{1}{4} \sqrt{10-2 \sqrt{5}}$ and $\sin \frac{2 \pi}{5}=\frac{1}{4} \sqrt{10+2 \sqrt{5}}$, so we obtain a contradiction. Hence we conclude that the only solution of (3) is the trivial one, meaning that $f_{A, \psi}$ is weakly mixing.

By Theorem 1 we then obtain that the group extension $f_{A, \psi}$ is 1-sided Bernoulli with respect to the Lebesgue (Haar) measure on $\mathbb{T}^{4}$.

Remarks. 1) Given the toral endomorphism $f_{A}$ from Theorem 2 (c), it follows from Corollary 11 that for a smooth perturbation $g$ of $f_{A}$ there exists a dense set of cocycles $\psi$ such that the extension $g_{\psi}$ is 1 -sided Bernoulli. In this way we can find a large class of examples of 1-sided Bernoulli group extensions.

2) Also from Theorem 2 it follows that expanding toral endomorphisms and their weak mixing group extensions do have generating Rohlin partitions (see [22], [5]).

\section{REFERENCES}

[1] R. L. Adler and P. C. Shields, Skew products of Bernoulli shifts with rotations, Israel J. Math., 12, 1972, 215-222. MR0315090 (47:3639)

[2] J. Ashley, B. Marcus and S. Tuncel, The classification of 1-sided Markov chains, Ergod. Th. and Dynam. Syst., 17, 1997, 269-295. MR.1444053 (98k:28021)

[3] H. Bruin and J. Hawkins, Rigidity of smooth one-sided Bernoulli endomorphisms, New York J. Math. 15, 2009, 1-33. MR2558792 (2011c:37013)

[4] Z. Coelho and W. Parry, Shift endomorphisms and compact Lie extensions, Bol. Soc. Bras. Mat., 29, 1, 1998, 163-179. MR1620172 (99d:28027)

[5] K. Dajani and J. Hawkins, Rohlin factors, product factors and joinings for $n$-to- 1 maps, Indiana Univ. Math. J., 42, 1993, 237-258. MR.1218714 (94h:28012)

[6] C. Hoffman, An endomorphism whose square is Bernoulli, Ergod. Th. and Dynam. Syst., 24, 2004, 477-494. MR2054190 (2005a:37006) 
[7] C. Hoffman and D. Rudolph, Uniform endomorphisms which are isomorphic to a Bernoulli shift, Annals of Math., 156, 2002, 79-101. MR.1935841 (2003i:37007)

[8] Y. Katznelson, Ergodic automorphisms of $\mathbb{T}^{n}$ are Bernoulli shifts, Israel J. Math., 10, 1971, 186-195. MR0294602(45:3672)

[9] I. Kornfeld and Y. Sinai, Chapters 1-3 in Dynamical Systems, Ergodic Theory and Applications, ed. Y. Sinai, Encycl. of Math. Sci., vol. 100, Springer Verlag, Berlin, Heidelberg, 2000. MR.1758456 (2001k:37004)

[10] R. Mane, Ergodic theory and differentiable dynamics, Springer Verlag, 1987. MR889254 (88c:58040)

[11] R. Mane, On the Bernoulli property for rational maps, Ergodic Th. and Dynam. Syst., 5, 71-88, 1985. MR782789 (86i:58082)

[12] E. Mihailescu, On some coding and mixing properties for a class of chaotic systems, Monatshefte fuer Math., 167, 2012, 241-255. MR2954528

[13] E. Mihailescu, Unstable directions and fractal dimensions for a family of skew products with overlaps, Math. Zeitschrift, 269, 2011, 733-750. MR2860262

[14] E. Mihailescu, Asymptotic distributions of preimages for endomorphisms, Ergod. Th. and Dynam. Systems, 31, 2011, 911-935. MR2794954 (2012e:37056)

[15] E. Mihailescu and M. Urbanski, Relations between stable dimension and the preimage counting function on basic sets with overlaps, Bull. London Math. Soc., 42, 2010, 15-27. MR2586963(2012c:37062)

[16] D. Ornstein, Bernoulli shifts with the same entropy are isomorphic, Advances in Math., 4, 1970, 337-352. MR0257322(41:1973)

[17] D. Ornstein and B. Weiss, Statistical properties of chaotic systems, Bull. AMS, 24, no. 1, 1991, 11-116. MR:1023980 (91g:58160)

[18] W. Parry, Automorphisms of the Bernoulli endomorphism and a class of skew products, Ergodic Th. and Dynam. Syst., 16, 1996, 519-529. MR1395050 (97h:28006)

[19] W. Parry, Skew products of shifts with a compact Lie group, J. London Math. Soc., 56, 1997, 395-404. MR 1489145 (99d:58108)

[20] W. Parry and M. Pollicott, Stability of mixing for toral extensions of hyperbolic systems, in Dynamical systems and related topics, Tr. Mat. Inst. Steklov, 216, Nauka, 1997, 354-363. MR.1632190 (99g:58094)

[21] W. Parry and P. Walters, Endomorphisms of a Lebesgue space, Bulletin AMS, 78, 1972, 272-276. MR0294604 (45:3674)

[22] V. A. Rohlin, Lectures on the entropy theory of transformations with invariant measure, Russian Math. Surv., 22, 1-54, 1967. MR0217258 (36:349)

[23] D. Rudolph, Classifying the isometric extensions of a Bernoulli shift, J. Analyse Math., 34, 1978, 36-60. MR:531270 (80g:28020)

[24] D. Ruelle, The thermodynamic formalism for expanding maps, Commun. in Math. Physics, 125, 239-262, 1989. MR1016871 (91a:58149)

[25] M. Shub, Endomorphisms of compact differentiable manifolds, American J. Math., 91, no. 1, 1969, 175-199. MR0240824 (39:2169)

[26] P. Walters, An introduction to ergodic theory (2nd edition), Springer, New York, 2000. MR648108 (84e:28017)

Institute of Mathematics of the Romanian Academy, P.O. Box 1-764, RO-014700, Bucharest, Romania

E-mail address: Eugen.Mihailescu@imar.ro

$U R L$ : www.imar.ro/ mihailes 